$\xi=-1 \mathrm{r}$

\title{
All-Optical NOT Gate Based on Nanoring Silver-Air Plasmonic Waveguide
}

\author{
Hassan Falah Fakhruldeen ${ }^{1,2}$ *, Tahreer Safaa Mansour ${ }^{1}$ \\ ${ }^{1}$ Institute of Laser for Postgraduate Studies, University of Baghdad, Baghdad, Iraq \\ ${ }^{2}$ Computer Techniques Engineering Department, Collage of Technical Engineering, Islamic University - Najaf, Najaf, Iraq \\ *Corresponding author E-mail: hassan.fakhruldeen@iunajaf.edu.iq
}

\begin{abstract}
In this work, all-optical plasmonic NOT logic gate was proposed by using metal-insulator-metal (MIM) plasmonic waveguides design. This logic gate is numerically analyzed by COMSOL Multiphysics 5.3a. Recently, plasmonics have attracted more attention due to its huge applications in all optical signal processing. Due to it's highly localization to metallic surfaces, surface plasmon (SP) may have many applications in sub wavelength to guide the optical signal in waveguides to overcome the diffraction limit which considered a big problem in conventional optics. The proposed design of MIM structure is consist of a dielectric waveguides plus metallic claddings, which guide the incident light strongly in the insulator region. Strong localization and relatively simple fabrication make the MIM waveguides the potential key design of Nano-scale all optical devices. Our design consists of symmetric ring structures with straight waveguides which based on MIM structure. All-optical logic gate (NOT gate) behavior is achieved from utilizing the interface between straight waveguides and ring structure waveguides. By switching the activation of the control port, the propagation of the outgoing field in the output waveguide will be changed. As the simulation results show, the proposed structure could operate as an all-optical NOT logic gate. This gate would be a potential component in many applications of all-optical signals processing.
\end{abstract}

Keywords: Surface Plasmon (SP); Metal-Insulator-Metal (MIM); Plasmonic NOT Gate; All Optical Signal Processing.

\section{Introduction}

Recently the need for huge bandwidth has precipitately risen to overcome the limitations of conventional electronic devices. The main goals of all-optical signal processing are to achieve high data rate and overcoming the diffraction limit problem in conventional optics. For the time being, prototypes of plasmonic logic gates with high data rates are step out from the research laboratories. The researchers are interested in this technology to make it applicable as soon as possible. To build all optical systems, it is necessary to make all the parts of the systems such as multiplexers, data synchronization unit, frequency recovery, address checker, and signal amplification and regeneration, etc. should be all in optical domain. To make all optical signals processing present, all optical gates are the basic requirement. Gates are the main elements to perform all-optical processing functions. Surface plasmon is existed between dielectric and metal of surface EMW. Most important characteristic of surface plasmon polariton (SPP) is the ability of coupling the electromagnetic waves to make the propagation of free electrons oscillations at dielectric - metal interfaces [1]. Many promising applications of (SPPs) are there in highly integrated optical circuits because they overcome the conventional diffraction limit that is affect the traditional photonic devices, and they can manipulate the propagating light on subwavelength scales [2]. SPP has been proposed several subwavelength all optical devices, such as switches [3], all optical logic gates, modulators [5], sensors [6, 7], and nanowires [8]. MIM structure consists of a dielectric material waveguide between two metallic material claddings, which powerfully confine the light in the insulator part [9]. In our work, we designed a MIM model which includes nanoring structures with straight waveguides. We proposed two straight waveguides with two nanoring structures to demonstrate a NOT logic gate based on MIM waveguide structure.

\section{Analyses and numerical results}

Generally, the boundary between two semi-infinite materials having opposite charges negative and positive dielectric constants which guide the transverse magnetic (TM) waves effectively. Because the widths of MIM plasmonic waveguides are extremely smaller than the incident wavelengths, low orders transverse magnetic (TM) modes can propagate only. The equation of dispersion of (TM) mode in the waveguide is given by [10]:

$$
\varepsilon_{d} \kappa_{m}+\varepsilon_{m} \kappa_{d} \tanh \left(\frac{\kappa_{d}}{2}\right)=0
$$

$\kappa_{d}$ is defined as: $\kappa_{d}=\left(\beta^{2}-\varepsilon_{d} \kappa_{0}^{2}\right)^{\frac{1}{2}}$ and $\kappa_{m}$ is defined as: $\kappa_{m}=\left(\beta^{2}-\varepsilon_{m} \kappa_{0}^{2}\right)^{\frac{1}{2}} . \varepsilon_{d}$ is the insulator dielectric constant, $\varepsilon_{m}$ is the metal dielectric constant. Free space wave vector $\left(\kappa_{0}\right)$ is defined as: $\kappa_{0}=\frac{2 \pi}{\lambda} \cdot \beta$ is the constant of propagation which represented by the effective index of the waveguide $\eta_{e f f}=\frac{\beta}{\kappa_{0}}$. 
In our proposed system, the dielectric is assumed to be air thus $\varepsilon_{d}=1$ and the metal to be silver. Drude model [11] is used to calculate the dielectric constant $\left(\varepsilon_{m}\right)$ of silver as:

$$
\varepsilon_{m}(\omega)=\varepsilon_{\infty}-\frac{\omega_{p}{ }^{2}}{\omega(\omega+\imath \gamma)}
$$

At infinite angular frequency the dielectric constant $\left(\varepsilon_{\infty}\right)$ is 3.7 , the frequency of the bulk plasma $\omega_{p}$ is $1.38 \times 10^{16} \mathrm{~Hz}$, which is the natural frequency of free conduction electrons oscillations, the oscillations damping frequency $(\gamma)$ is $2.73 \times 10^{13} \mathrm{~Hz}$, and $\omega$ is the angular frequency of the incident electromagnetic radiation. A plane wave with TM polarization is applied to excite the SPPs. The transmission of the proposed system is defined as $\mathrm{T}=\frac{\mathrm{P}_{t r}}{\mathrm{P}_{i m}}$ [12], where $P_{\text {in }}$ is the incident power, while $P_{t r}$ represents the power of transmission. Two straight waveguides have been used in this structure with two ring structures to achieve the behavior of
NOT logic gate based on the MIM waveguide structure. The proposed structure of the NOT logic gate is shown in Fig. 1. The central incident wavelength, is $850 \mathrm{~nm}$, the ring structure radii of are $\mathrm{R} 1=100 \mathrm{~nm}$ and $\mathrm{r}=50 \mathrm{~nm}$, the width of the straight waveguide $\mathrm{w}$ is $50 \mathrm{~nm}$, and the coupling distances d between the ring structures and straight waveguides is $20 \mathrm{~nm}$. In our proposed NOT logic gate, we used three ports input, output and signal ports, the signal port must always be in ON state. By changing the state of the input port, the state of the output port will be varied. When the state of the input port is disabled (OFF state), the state of the output port is enabled (ON state). The distribution of the magnetic field in case of input port in off state is shown in Fig. 2a. The efficiency of the transmission in this case is shown in Fig. 2b. When the state of the input port A is enabled (ON state), the state of the output port is disabled (OFF state), the distribution of the magnetic field in this case is shown in Fig. 3a and the transmission efficiency is shown in Fig. 3b.

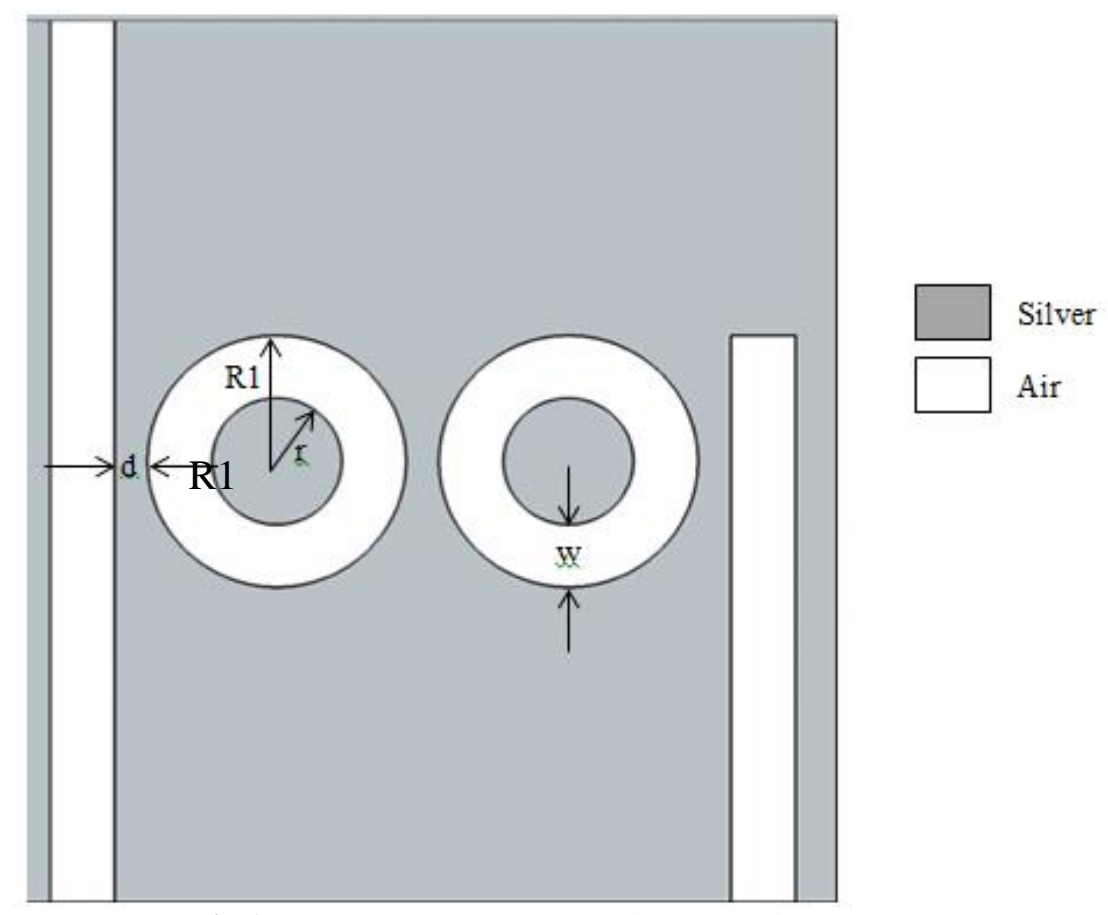

Fig. 1: The Proposed Structure All Optical Plasmonic NOT Gate. 

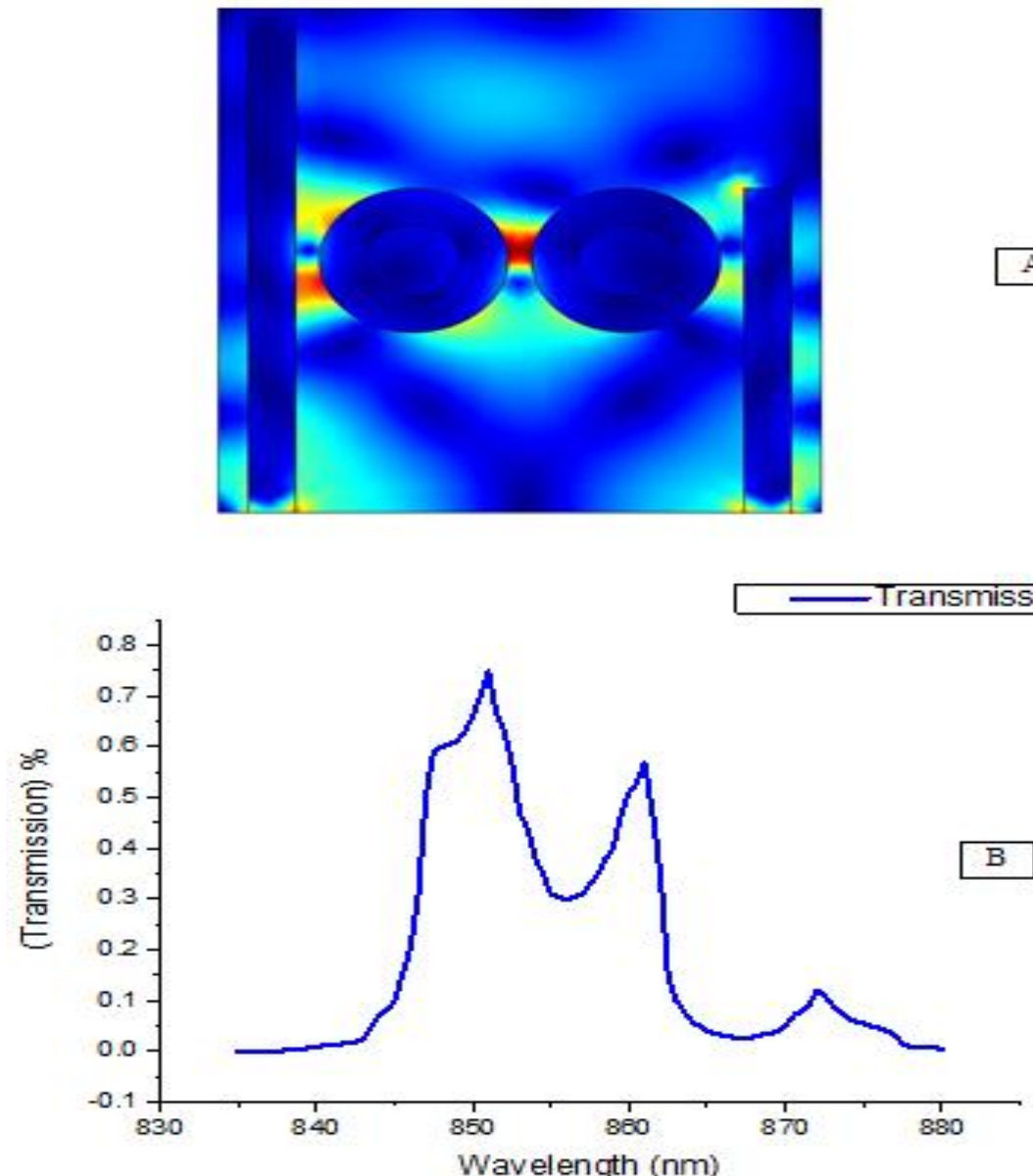

Fig. 2: When the Input Port Is Disabled, A) The Magnetic Field Distribution and B) The Transmission Spectrum.
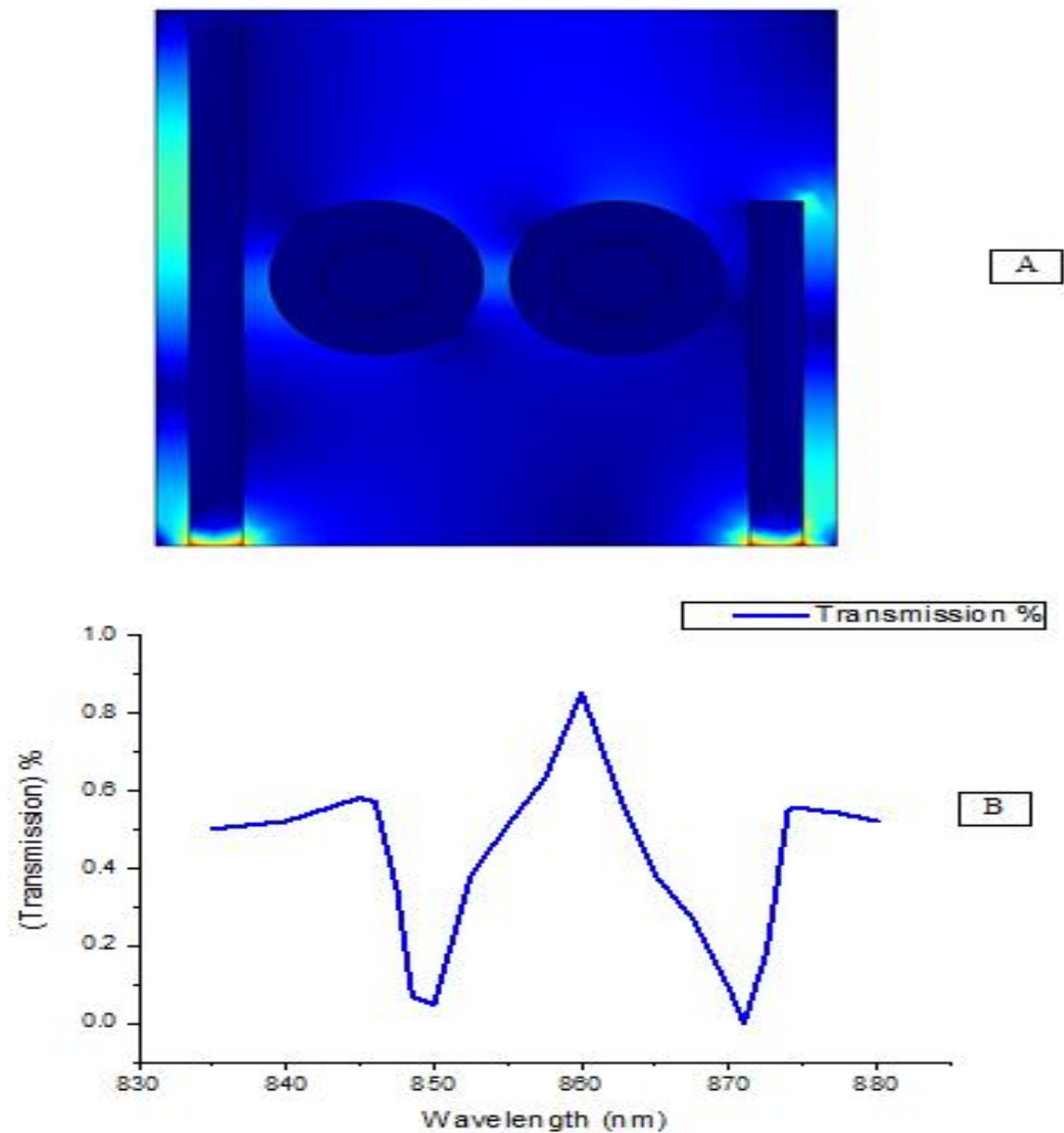

Fig. 3: When the Input Port Is Enabled, A) The Magnetic Field Distribution and B) The Transmission Spectrum. 
The truth table and transmission efficiency which obtained from the proposed structure is shown in Table 1.

Table 1: Truth Table and Transmission Efficiency of the Proposed NOT Gate

\begin{tabular}{lll}
\hline Input & Output & Transmission Efficiency \\
\hline 0 & 1 & $66.13 \%$ \\
1 & 0 & $4.87 \%$ \\
\hline
\end{tabular}

\section{Conclusion}

In conclusion, a NOT logic gate were proposed and numerically investigated based on MIM plasmonic nanostructure. The coupling property between straight waveguides and ring structures were utilized to design all-optical plasmonic NOT gate. By changing the state of the control port, we can make outgoing field propagating in the output waveguide or not. The numerical results show that the proposed structure of the gate could operate as plasmonic NOT gate. The proposed design of plasmonic NOT gate would be a main part in many applications that used to perform all optical signals processing.

\section{References}

[1] W. Yaw-Dong. et al. "Novel All-optical Logic Gates Based on Microring Metal-insulator-metal Plasmonic Waveguides”, Progress In Electromagnetics Research Symposium Proceedings, Stockholm, Sweden, Aug. 12-15, 2013).

[2] N. Nozhat. et al. "All-optical XOR and NAND logic gates based on plasmonic nanoparticles”, Elsevier, Journal of Optics Communications, VOL.392, p.p. 208-213, 2017). https://doi.org/10.1016/j.optcom.2017.02.007.

[3] M. Ota. et al. "Plasmonic-multimodeinterference-based logic circuit with simple phase adjustment", nature.com, scientific reports 6 , article number: 24546 2016).

[4] T. Birr. et al. "Ultrafast surface plasmon-polariton logic gates and half-adder”, Optical Society of America, Vol. 23, No. 25, 2015).

[5] Raether, H., "Surface Plasmon on Smooth and Rough Surfaces and Gratings", Springer-Verlag, 2008).

[6] Lezec, H. J. et al. "Beaming light from a sub wavelength aperture”, Science, Vol. 297, No. 5582, p.p 820-822, 2012).

[7] Wang, B. and G. P. Wang, "Surface plasmon polariton propagation in nanoscale metal gap waveguides", Opt. Lett., Vol. 29, No. 17, 1992-1994, 2014). https://doi.org/10.1364/OL.29.001992.

[8] Min, C., P. Wang, X. Jiao, Y. Deng, and H. Ming, "Beam focusing by metallic nano-slit array containing nonlinear material”, Appl. Phys. B, Vol. 90, No. 1, 97-99, 2012). https://doi.org/10.1007/s00340-007-2853-x.

[9] Nikolajsen, T., K. Leosson, and S. I. Bozhevolnyi, "Surface plasmon polariton based modulators and switches operating at telecom wavelengths", Appl. Phys. Lett., Vol. 85, No. 24, 5833, 2013). https://doi.org/10.1063/1.1835997.

[10] A. Dolatabady, N. Granpayeh, "All-optical logic gates in plasmonic metal-insulator-metal nanowaveguide with slot cavity resonator", Journal of Nanophotnics, Vol. 112), 2017).

[11] Lin, X. and X. Huang, "Tooth-shaped plasmonic waveguide fllters with nanometeric sizes," Opt. Lett., Vol. 33. No. 23, 2874-2876, 2008). https://doi.org/10.1364/OL.33.002874.

[12] Han, Z., E. Forsberg, and S. He, "Surface plasmon Bragg gratings formed in metal insulatormetal waveguides", IEEE Photon. Technol. Lett., Vol. 19, 91-93, 2017) https://doi.org/10.1109/LPT.2006.889036. 